\title{
Synthesis of Hydrogen Network
}

\author{
Chun Deng ${ }^{1} \cdot$ Yongzhong Liu $^{2} \cdot$ Zuwei Liao $^{3}$
}

Received: 19 April 2018 / Revised: 20 April 2018 / Accepted: 21 April 2018 / Published online: 28 May 2018

(C) Springer Nature Singapore Pte Ltd. 2018

The theme of this special section is "Synthesis of Hydrogen Network." A call for papers for the special section was announced on Feb 4th, 2017. A total of five papers were accepted for publication after the peer review process. The papers come from outstanding research groups from China and France. The papers broadly fall within two general themes, process synthesis and hydrogen supply chain (HSC). A brief review of these papers is given as follows.

\section{Papers on Process Synthesis}

Four papers in this special section report the latest work on synthesis of hydrogen network. These include the placement of purifier (Lou et al. 2017), strategies of merging compressors (Liang and Liu 2017), flowrate targeting for hydrogen network with single intermediate header (Deng et al. 2018), and hydrogen partial pressure correction model (Li and Zhang 2018).

Purifiers are widely used in refinery to upgrade the hydrogen sources for further utilization. Purifier models such as PSA and membrane separation have nonlinear characteristics, and the global optimal solution cannot be easily guaranteed. To address the problem, a mixed integer linear programming model is proposed to obtain a minimum flowrate of hydrogen

Chun Deng

chundeng@cup.edu.cn

Yongzhong Liu

yzliu@mail.xjtu.edu.cn

Zuwei Liao

liaozw@zju.edu.cn

1 State Key Laboratory of Heavy Oil Processing, College of Chemical Engineering, China University of Petroleum, Beijing, China

2 Department of Chemical Engineering, Xi'an Jiaotong University, Xi'an, Shaanxi 710049, China

3 State Key Laboratory of Chemical Engineering, College of Chemical and Biological Engineering, Zhejiang University,, Beijing, China utility and the minimum inlet flowrate of purifier (Lou et al. 2017). It avoids solving a nonlinear model and ensures the minimum fresh hydrogen global optimality. Case studies indicate that the method is effective for both hydrogen and water networks.

To reduce the number of compressors and capital cost, Liang and Liu (2017) proposed two strategies of merging compressors, namely, the hydrogen sources-oriented compressors merging strategy and hydrogen sinks-oriented compressors merging strategy. The results of one case study show that the number and the capital cost of compressors can be reduced. The flexibility analysis shows that the operating flexibility of the network after merging compressors can be improved.

In the work of Deng et al. (2018), the generalized improved problem table is introduced for flowrate targeting of hydrogen networks with single intermediate header and up to one purifier. Two scenarios for direct reuse/recycling and one scenario for purification reuse/recycling are analyzed to illustrate the proposed approach. The results show that the total numbers of connections for networks with an intermediate header are almost identical with those for the conventional network without the intermediate header. However, the total number of direct connections between hydrogen sources and sinks would be reduced. It may increase the network resistance to flowrate and purity fluctuations that often occur during plant operation, and it will be addressed in the future work.

Hydrogen partial pressure was estimated by Dalton's law which is not suitable to real gas especially in higher pressure situation. To overcome the limitation, Li and Zhang (2018) introduced the theorem of corresponding states and compressibility factor to calculate hydrogen partial pressure accurately. Based on relative concentration and hydrogen partial pressure properties for hydrogen streams, a nonlinear programming model for the minimization of total exergy including fresh hydrogen and compression work is proposed. Case studies show that hydrogen partial pressure calculated by introduced thermodynamic principles is more accurate and it would save compression work. 


\section{Papers on Hydrogen Supply Chain}

In the work of Ochoa Robles et al. (2017), a model of the hydrogen supply chain has been developed through a mixed integer linear programming formulation. The sensitivity analysis of six key parameters (demand, capital change factor, storage and production capital costs, learning rate, and unit production cost) is performed. The demand is identified as the most significant parameter that strongly conditions the total daily cost. It is pointed out that the uncertainty of demand is a critical issue for the future HSCs.

Acknowledgments The editors would like to acknowledge the excellent support of Frank Franco in the PIOS editorial office in managing this special section. Efforts for peer reviewing from all reviewers are greatly appreciated and ensure a high-quality special section is delivered.

\section{References}

Deng C, Zhou Y, Zhu M, Lee J-Y, Foo DCY (2018) Improved problem table for targeting hydrogen network with single intermediate header. Process Integr Optim Sustain. https://doi.org/10.1007/s41660017-0031-0

Li J, Zhang Q (2018) Thermodynamic principle-based hydrogen partial pressure correction for optimization of refinery hydrogen networks. Process Integr Optim Sustain. https://doi.org/10.1007/s41660-0180035-4

Liang X, Liu Y (2017) Strategies of merging compressors for capital cost reduction in hydrogen networks synthesis. Process Integr Optim Sustain. https://doi.org/10.1007/s41660-017-0017-y

Lou Y, Liao Z, Wang J, Yang Y (2017) MILP model for the nonlinear problem of optimal purifier placement. Process Integr Optim Sustain. https://doi.org/10.1007/s41660-017-0018-x

Ochoa Robles J, De-León Almaraz S, Azzaro-Pantel C (2017) Design of experiments for sensitivity analysis of a hydrogen supply chain design model. Process Integr Optim Sustain. https://doi.org/10.1007/ s41660-017-0025-y 